\title{
Hybrid of HMM and fuzzy logic for handwritten character recognition
}

\begin{abstract}
This paper presents a hybrid approach of HMM and Fuzzy Logic in the field of handwritten character recognition. Fuzzy Logic is used in the recognition phase while HMM is used in the process of extracting features for the preparation of linguistic variables of the fuzzy rules. Experimental results from a few sample images give a reasonable recognition rate on a more challenging database of lower-case handwritten characters. This proved the proposed hybrid of the two techniques are compatible and can be used to complement each other effectively.
\end{abstract}

